\title{
Donor human leukocyte antigen-G single nucleotide polymorphisms are associated with post-lung transplant mortality
}

\author{
Julieta Lazarte ${ }^{1,2}$, Jin $\mathrm{Ma}^{3}$, Tereza Martinu ${ }^{1}$, Liran Levy ${ }^{1}$, William Klement ${ }^{1}$, \\ Matthew White ${ }^{1}$, Jacob Pelling ${ }^{1}$, Zehong Guan ${ }^{1}$, Sassan Azad ${ }^{1}$, Jussi Tikkanen ${ }^{1}$, \\ Vivek Rao ${ }^{2}$, George Tomlinson ${ }^{3}$, Diego Delgado ${ }^{2}$, Shaf Keshavjee ${ }^{1}$ and \\ Stephen C. Juvet ${ }^{1}$
}

Affiliations: ${ }^{1}$ Toronto Lung Transplant Program, Toronto General Hospital, Toronto, ON, Canada. ${ }^{2}$ Cardiac Transplant Program, Toronto General Hospital, Toronto, ON, Canada. ${ }^{3}$ University Health Network, University of Toronto, Toronto, Canada.

Correspondence: Stephen C. Juvet, Toronto General Hospital, 200 Elizabeth Street, Room 11 PMB 126, Toronto, ON, M5G 2C4, Canada. E-mail: stephen.juvetđuhn.ca

@ERSpublications

This largest-ever study on HLA-G SNPs and their relationship to transplant outcomes recognises the role of lung donor HLA-G SNPs on allograft HLA-G expression and outcomes post lung transplantation, a factor previously ignored http://bit.ly/2VZURhm

Cite this article as: Lazarte J, Ma J, Martinu T, et al. Donor human leukocyte antigen-G single nucleotide polymorphisms are associated with post-lung transplant mortality. Eur Respir J 2019; 54: 1802126 [https:// doi.org/10.1183/13993003.02126-2018].

ABSTRACT Human leukocyte antigen (HLA)-G is a non-classical HLA that inhibits immune responses. Its expression is modified by single nucleotide polymorphisms (SNPs), which are associated with transplant outcomes. Our aim was to investigate the association of donor and recipient HLA-G SNPs with chronic lung allograft dysfunction (CLAD) and mortality after lung transplantation.

In this single-centre study, we examined 11 HLA-G SNPs in 345 consecutive recipients and 297 donors of a first bilateral lung transplant. A multivariable Cox proportional hazards model assessed associations of SNPs with death and CLAD. Transbronchial biopsies (TBBx) and bronchoalveolar lavage (BAL) samples were examined using quantitative PCR, ELISA and immunofluorescence.

Over a median of 4.75 years, 142 patients (41\%) developed CLAD; 170 (49\%) died. Multivariable analysis revealed donor SNP +3142 (GG+CG versus CC) was associated with increased mortality (hazard ratio 1.78, 95\% CI 1.12-2.84; $\mathrm{p}=0.015)$. In contrast, five donor SNPs, -201(CC), -716(TT), -56(CC), $\mathrm{G}^{\star} 01: 03(\mathrm{AA})$ and $14 \mathrm{bp}$ INDEL, conferred reduced mortality risk. Specific donor-recipient SNP pairings reduced CLAD risk. Predominantly epithelial HLA-G expression was observed on TBBx without rejection. Soluble HLA-G was present in higher concentrations in the BAL samples of patients who later developed CLAD.

Specific donor SNPs were associated with mortality risk after lung transplantation, while certain donorrecipient SNP pairings modulated CLAD risk. TBBx demonstrated predominantly epithelial, and therefore presumably donor-derived, HLA-G expression in keeping with these observations. This study is the first to demonstrate an effect of donor HLA-G SNPs on lung transplantation outcome. 


\section{Introduction}

Median survival after lung transplantation (LT) is limited to 5.7 years by chronic lung allograft dysfunction (CLAD) [1, 2]. CLAD is progressive, with limited preventative and therapeutic options [3]; re-transplantation is available only for selected patients [4]. Identification of donor and recipient factors present at the time of transplantation that may modify graft-damaging alloimmune and inflammatory responses is therefore highly desirable.

Human leukocyte antigen (HLA)-G is a non-classical class Ib protein that exists in membrane-bound and various soluble isoforms due to alternative splicing $[5,6]$. It is expressed in placenta, leukocytes and allograft parenchymal cells [7,8], where it inhibits lymphocytes [9], promotes the induction of regulatory cells $[7,10]$ and inhibits rejection $[11,12]$. Unlike most HLA molecules, it exhibits limited variation in the protein-coding region, but its expression is modified by single nucleotide polymorphisms (SNPs), many of which are located in regulatory upstream and downstream elements of the HLA-G locus [13].

Higher HLA-G expression in lung tissue has been observed in LT patients free of rejection or CLAD [8]. In contrast, elevated soluble HLA-G (sHLA-G) concentrations in bronchiolar lavage (BAL) fluid has been associated with acute rejection in patients who subsequently developed CLAD [14]. Lung recipients with specific SNPs had lower serum sHLA-G concentrations and increased risk of CLAD [15], suggesting that systemic but not BAL fluid HLA-G expression may attenuate CLAD risk.

To date, no studies have examined the influence of donor HLA-G on CLAD and mortality after LT. This is an important question because donor-derived graft cells express HLA-G $[8,16]$. Because HLA-G is expressed by recipient leukocytes, different combinations of donor and recipient HLA-G expression may influence outcomes. We previously found that both donor and recipient HLA-G SNPs modulated risk for chronic allograft vasculopathy after heart transplantation [17]. Therefore, our goal was to determine the effect of donor and recipient HLA-G SNPs on CLAD and mortality after LT.

\section{Methods}

Patients and follow-up

Between January 1, 2007, and December 31, 2011, 473 patients underwent LT in the Toronto Lung Transplant Program (TLTP; figure 1a). Recipients aged $<18$ years at transplant $(n=18)$, or who underwent single LT $(n=67)$, multi-organ transplant $(n=9)$ or lung re-transplant $(n=14)$ were excluded. The remaining patients $(n=345)$ who underwent a first bilateral lung transplant and their corresponding donors $(n=345)$ were eligible for inclusion in this single-centre retrospective study. From these, we excluded one recipient lost to follow-up, and 19 recipients and 48 donors with missing or insufficient DNA for genotyping. In total, 345 recipients and 297 donor-recipient pairs were included. Recipients' demographic and clinical data were collected from electronic medical records. Patients were treated with azathioprine (or mycophenolate for patients with anti-HLA antibodies), a calcineurin inhibitor (cyclosporine or tacrolimus) and prednisone. Beginning in 2008, patients with pre-transplant sensitisation received intra- and post-operative plasmapheresis, intravenous immunoglobulin replacement and anti-thymocyte globulin [18]. Follow-up included regular pulmonary function tests, thoracic imaging, and bronchoscopies. Donor and recipient DNA was obtained from the University Health Network HLA laboratory. The study protocol was approved by the institutional research ethics board.

CLAD and mortality were recorded for each recipient until March 22, 2016 (median follow-up 4.75 years). Graft survival was defined as freedom from death or re-transplantation. CLAD was determined according to International Society for Heart and Lung Transplantation (ISHLT) criteria (forced expiratory volume in $1 \mathrm{~s}(\mathrm{FEV} 1) \leqslant 80 \%$ of baseline for $\geqslant 3$ weeks; absence of another specific cause) using a semi-automated pulmonary function test algorithm $[19,20]$. All CLAD diagnoses were confirmed by a respiratory physician (SCJ, JT or TM) blinded to HLA-G data.

\section{Selection of patients for HLA-G measurement in BAL samples}

Beginning in 2011, the TLTP routinely biobanked BAL supernatants and cell pellets from all consenting recipients. Our cohort included 82 patients who underwent LT in 2011 (figure 1b). To examine the relationship between BAL sHLA-G and CLAD, we compared patients who developed CLAD within 3 years $(n=25)$ with those who maintained stable lung function for at least 4 years $(n=43)$ and who had at least one available sample from their 3,6 or 9 month surveillance bronchoscopies. To facilitate a comparison between patients with early CLAD (within 3 years) and later-onset CLAD (after 4 years), we excluded patients diagnosed with CLAD between 3 and 4 years post transplant $(n=6)$ and patients who died between 3 and 4 years post transplant $(n=3)$. We also excluded patients with no stored BAL sample $(\mathrm{n}=5)$. There were 23 missing samples. 
a) SNP analysis

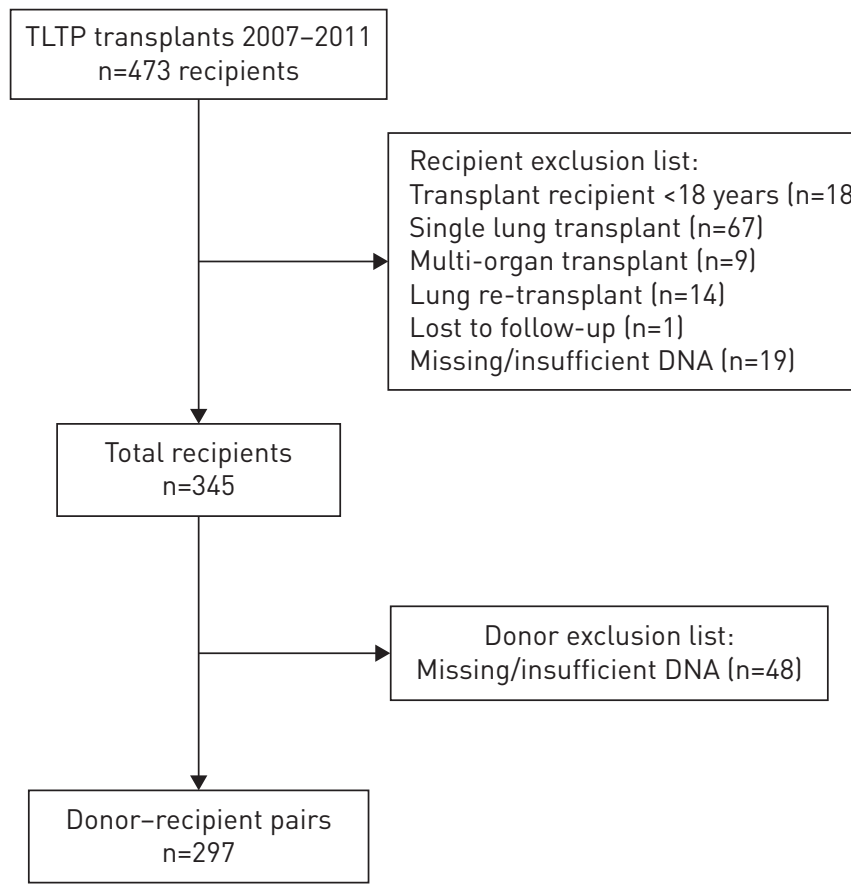

b) BAL sHLA-G analysis

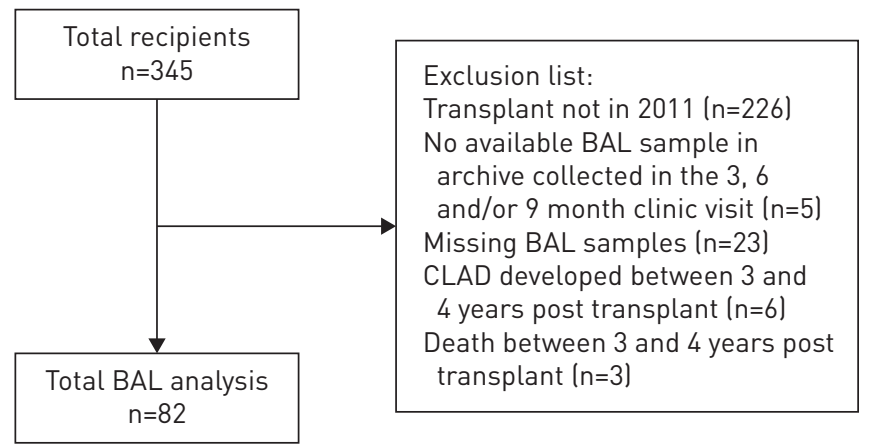

c) TBBx HLA-G analysis

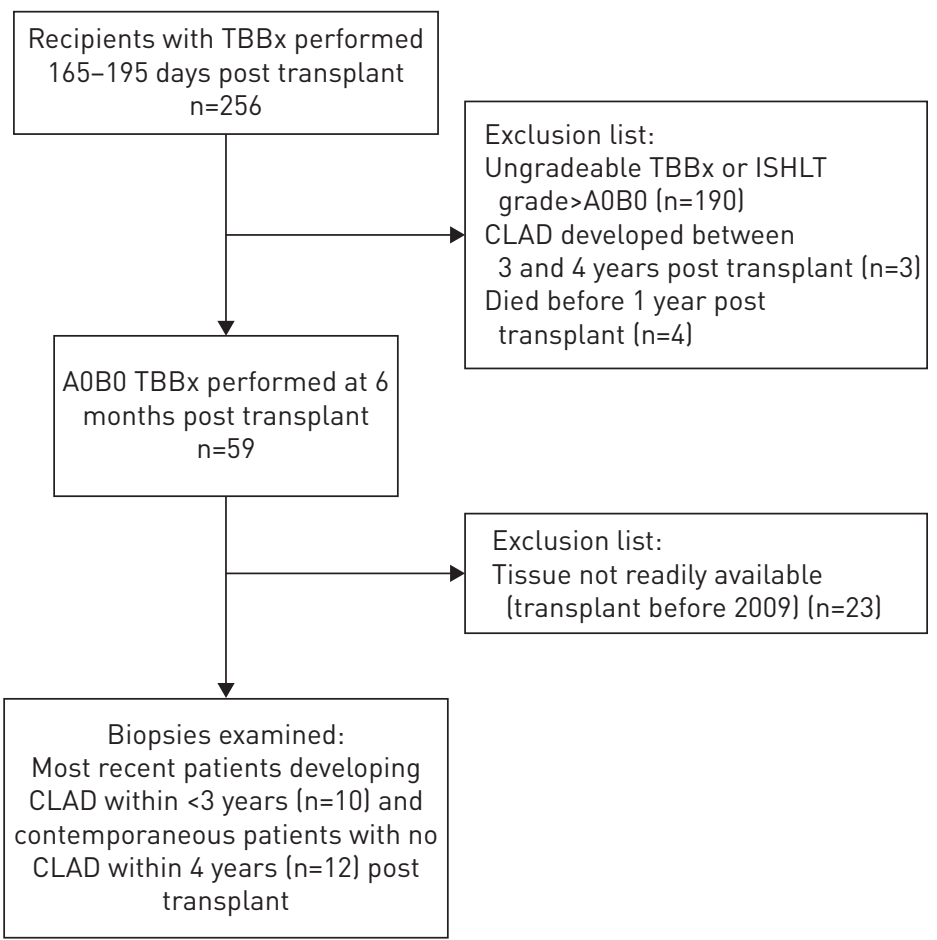

FIGURE 1 a) Flow chart depicting inclusion and exclusion of lung allograft donors and recipients in this retrospective cohort study. b) Selection of patients for analysis of bronchoalveolar lavage (BAL) samples. c) Selection of patients for analysis of transbronchial biopsy (TBBx) samples. SNP: single nucleotide polymorphism; TLTP: Toronto Lung Transplant Program; sHLA-G: soluble human leukocyte antigen-G; CLAD: chronic lung allograft dysfunction; ISHLT: International Society for Heart and Lung Transplantation.

\section{Selection of patients for transbronchial biopsy immunofluorescence}

Our cohort included 256 patients with transbronchial biopsies (TBBx) performed between 165 and 195 days (i.e. 6 months) post transplant. Of these, 59 had an ISHLT grade of A0B0. We excluded patients who developed CLAD between 3 and 4 years post transplant, those who died before 1 year post transplant and those for whom tissue was not readily available. We selected the 10 most recent patients developing CLAD within 3 years and 12 contemporaneous patients free of CLAD for at least 4 years post transplant (figure 1c). The biopsies from these patients had a median of five (range four to nine) evaluable parenchymal fragments. 
Laboratory analyses

Details of SNP analysis, HLA-G ELISA, PCR and immunofluorescence are in the supplementary material.

\section{Statistical analysis}

Recipient and donor genotypes were analysed according to recessive effect, dominant effect, allele effect and pairing effects to examine associations with death or CLAD (details can be found in the supplementary material). A Cox proportional hazards model was used to evaluate the association between genotype and mortality, adjusted for cytomegalovirus (CMV) status. Both the Cox model (censoring patients at death or CLAD onset) and the Fine and Gray model (treating death as a competing risk for CLAD) were used. BAL sHLA-G concentrations in individual patients over time were analysed as a time-dependent covariate for CLAD using a Cox proportional hazards model adjusted for CMV status. We also ran an analysis in which missing BAL sHLA-G concentrations were interpolated with the average of other observed values, because they tended to be stable over time. All statistical analyses were performed using R version 3.5.1 (R Foundation for Statistical Computing, Vienna, Austria).

\section{Results}

Recipient and donor characteristics and outcomes

Recipient and donor demographics are shown in table 1 . Mean recipient and donor ages were $50 \pm 14$ and $47 \pm 17$ years, respectively. Median follow-up time was 4.75 years (range $0.01-9.13$ years). The median overall survival time was 6.37 years and at last follow-up 170 (49\%) had died (supplementary figure S1). The median CLAD-free survival time was 5.65 years and 142 (43\%) patients had CLAD at the end of the study (supplementary figure S2). These data compare favourably with the ISHLT Registry [1].

\section{TABLE 1 Recipient, donor and pre-transplant characteristics of the study cohort}

\section{Characteristics}

Recipient characteristics

Age at transplant years

Male sex

Primary diagnosis

COPD

Cystic fibrosis

Interstitial lung disease

Pulmonary vascular disease Other ${ }^{\#}$

Donor characteristics

Age of donor years

Male sex

Cause of death

Non-heart beating/DCD

Pre-transplant characteristics

CMV match status

Donor -, Recipient -

Donor -, Recipient +

Donor +, Recipient -

Donor +, Recipient +

PRA status + DSA status

PRA 0, DSA 0

PRA +, DSA 0

PRA +, DSA +

PRA NA, DSA NA

HLA DQ mismatch

No mismatch

1 Mismatch

2 Mismatch

Acute cellular rejection (ISHLT grade A1 or greater at any time)
Subjects $n$

345

$50 \pm 14$

$193(56)$

$107(31)$

79 (23)

105 (30)

$16(5)$

$38(11)$

297

$47 \pm 17$

143 (48)

$26(9)$

345

80 (23)

98 (28)

$89(26)$

78 (23)

345

$143(41)$

65 (19)

43 (12)

94 (27)

212

25 (12)

27 (13)

$160(76)$

176 (70)

Data are presented as $\mathrm{n}(\%)$ or mean \pm SD, unless otherwise indicated. COPD: chronic obstructive pulmonary disease; DCD: donor after cardiac death; CMV: cytomegalovirus; PRA: panel-reactive antibodies; DSA: donor-specific antibody; NA: not applicable; HLA: human leukocyte antigen; ISHLT: International Society for Heart and Lung Transplantation. \#: bronchiolitis obliterans, bronchiectasis, bronchioloalveolar carcinoma, lymphangioleiomyomatosis, Langerhans cell histiocytosis. 
Donor and recipient HLA-G SNPS

Recipient and donor allele frequencies for the investigated polymorphisms are shown in supplementary table S1. With the exception of SNP +3196, all donor and recipient SNP allele frequencies were in HardyWeinberg equilibrium. A total of 297 donor-recipient pairs (86\%) were available for analysis.

Donor HLA-G SNPS modify the risk of death after LT

Clinical variables known to influence post-lung transplant mortality and CLAD were tested in univariate analyses (supplementary tables S2 and S3). Primary CMV mismatch (donor positive/recipient negative) was the only significant variable and was therefore included in the multivariate analysis. Donor SNP +3142 (GG or CG) allele was identified as a risk factor for mortality (hazard ratio (HR) 1.78, 95\% CI 1.12-2.84, $\mathrm{p}=0.015$; table 2 and figure 2a). Additionally, five donor SNPs conferred decreased mortality risk (table 2). We also identified SNP +3142(GG) allele as a risk factor for reduced CLAD-free survival (HR 1.37, 95\% CI 1.01-1.86, $\mathrm{p}=0.042$; figure $2 \mathrm{~b}$ and supplementary tables $\mathrm{S} 4-\mathrm{S} 6$ ). We did not observe any significant effects when death was treated as a competing risk for CLAD (supplementary tables S7-S9). Finally, we did not find any statistically significant associations between recipient HLA-G SNPs and CLAD or survival in this cohort (not shown). These observations indicate that donor genetic variants in HLA-G can modify long-term outcome after LT.

\section{Specific donor and recipient HLA-G SNP pairings reduce the risk of CLAD after LT}

We next examined the influence of HLA-G SNPs on CLAD development. We adjusted the multivariate analysis for primary CMV mismatch. Donor-recipient pairing at SNP -201(CC-CT alleles) and donorrecipient pairing at SNP -716(GT-TT alleles) both independently conferred a reduced risk for CLAD (HR 0.45, 95\% CI 0.21-0.95, $\mathrm{p}=0.036$; table 3). These observations reveal that while donor HLA-G SNPs modified the risk of mortality after LT, the risk of CLAD is mitigated by specific donor-recipient HLA-G SNP pairings.

Higher SHLA-G levels in BAL fluid are associated with an increased risk of CLAD

We observed higher sHLA-G concentrations in the BAL supernatants of patients who had CLAD within 3 years compared with patients who did not develop CLAD by 4 years (figure $3 \mathrm{a}-\mathrm{c}$ ). Concentrations of sHLA-G in BAL fluid decreased over time in the latter group compared to the CLAD patients, with differences becoming more pronounced at 6 and 9 months post transplant $(p=0.05$ and $p=0.03$ ). Considering sHLA-G in the BAL fluid as a time-dependent variable, we found that higher concentrations were associated with increased CLAD risk, even after adjustment for CMV status (HR 1.21 for every 10 international units per $\mathrm{mL}, \mathrm{p}=0.03$; table 4 ).

We quantified HLA-G transcripts in cell pellets from the same BAL samples. These did not have any bearing on CLAD either when considered at each time point (figure $3 \mathrm{~d}-\mathrm{f}$ ) or when treated as a time-dependent risk factor (data not shown). Moreover, we found no association between HLA-G transcript abundance and sHLA-G protein in the same sample (figure $3 \mathrm{~g}$ ). In this small subset of patients, these observations suggest that sHLA-G protein concentrations in BAL fluid are not directly related to the level of HLA-G gene transcription in BAL cells, but that higher levels may be associated with an increased risk of subsequent CLAD.

TABLE 2 Multivariable analysis for time to death

\begin{tabular}{lccc} 
Variable & Hazard ratio (95\% CI) & p-value & $\mathbf{R}^{2}$-value \\
\hline SNP 3142 (donor) (GG+CG versus CC) & $1.78(1.12-2.84)$ & 0.015 & 0.049 \\
(GG versus CG+CC) & $1.49(1.06-2.10)$ & 0.021 & 0.044 \\
(GG versus CC) & $2.12(1.27-3.53)$ & 0.004 & 0.057 \\
SNP 201 (donor) (CC versus CT+TT) & $0.63(0.41-0.96)$ & 0.032 & 0.044 \\
(CC versus TT) & $0.58(0.35-0.95)$ & 0.032 & 0.045 \\
SNP 716 (donor) (TT versus GT+GG) & $0.63(0.41-0.96)$ & 0.032 & 0.044 \\
(TT versus GG) & $0.58(0.35-0.95)$ & 0.032 & 0.045 \\
SNP 56 (donor) (CC versus CT+TT) & $0.53(0.32-0.90)$ & 0.017 & 0.043 \\
G*01:03 (donor) (AA versus AT+TT) & $0.57(0.34-0.96)$ & 0.034 & 0.041 \\
14BP INDEL (donor) (DEL versus INDEL+INS) & $0.67(0.46-0.97)$ & 0.032 & 0.044 \\
\hline
\end{tabular}

Adjusted for cytomegalovirus status. SNP: single nucleotide polymorphism. 

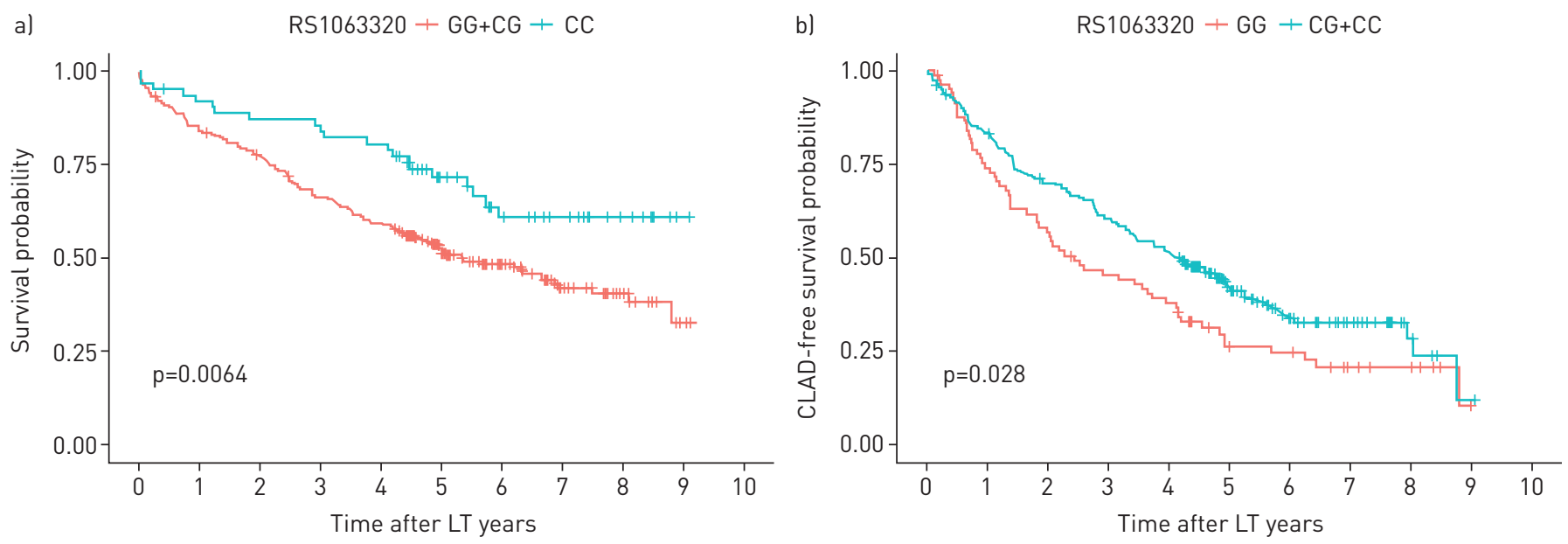

\begin{tabular}{|c|c|c|c|c|c|c|c|c|c|c|c|}
\hline \multirow[b]{2}{*}{$\mathrm{GG}+\mathrm{CG}$} & \multicolumn{11}{|c|}{ Number at risk } \\
\hline & 236 & 197 & 180 & 153 & 137 & 95 & 69 & 36 & 20 & 3 & 0 \\
\hline CC & 63 & 56 & 53 & 52 & 49 & 31 & 21 & 15 & 8 & 1 & 0 \\
\hline
\end{tabular}

Number at risk
\begin{tabular}{|cccccccccccccc|}
\hline CG+CC & 62 & 46 & 36 & 30 & 15 & 14 & 8 & 5 & 0 & 0 \\
203 & 168 & 139 & 120 & 102 & 62 & 31 & 20 & 6 & 1 & 0 \\
\hline
\end{tabular}

FIGURE 2 a) Kaplan-Meier curves depicting the probability of survival of lung transplantation (LT) recipients according to donor RS1063320 (single nucleotide polymorphism (SNP) +3142) genotype. Blue line: donor CC allele; red line: donor GG or CG alleles for SNP +3142. Lung donor GG or GC genotype at SNP +3142 increased the risk for mortality compared with donor CC genotype ( $p=0.0064)$. b) Kaplan-Meier curves depicting the probability of chronic lung allograft dysfunction (CLAD)-free survival of LT recipients according to donor RS1063320 (SNP +3142) genotype. Blue line: donor CC or GC alleles; red line: donor GG allele at SNP +3142. Lung donor GG genotype at SNP +3142 decreased CLAD-free survival probability compared with donor CC genotype ( $\mathrm{p}=0.028)$.

\section{Allograft epithelium exhibits greater HLA-G expression than allograft leukocytes}

We examined HLA-G expression on donor- and recipient-derived cells in the allograft. In contrast to BRUGIERE et al. [8], we did not observe differences in HLA-G staining between patients with and without CLAD (data not shown) in this small subset. However, we noted much stronger HLA-G staining on epithelium than on leukocytes in the same biopsy (figure 4a-c), suggesting that donor-derived HLA-G expression continues post transplant and likely represents the major source of HLA-G in the lung allograft. Additionally, biopsies from recipients whose donors had the CC genotype at SNP +3142 exhibited a higher HLA-G staining intensity than biopsies from recipients whose donors had the CG or GG genotype $(\mathrm{p}=0.04$; figure $4 \mathrm{~d})$, consistent with our observation that donor SNP $+3142(\mathrm{CC})$ genotype is protective against mortality following LT.

\section{Discussion}

HLA-G expression is dictated by genetic and environmental factors [7], and a better understanding of these determinants will help us comprehend HLA-G's influence on allograft outcomes. Our data suggest that donor HLA-G polymorphisms are associated with a stronger influence on LT outcome than recipient ones. However, specific donor-recipient SNP pairings modulated CLAD risk. Previous studies have shown that the G allele of SNP +3142 is associated with lower HLA-G production, due to enhanced targeting by microRNAs [21]. Expression of HLA-G molecules by allograft epithelium is under the control of the donor HLA-G genotype, and in a small sample of biopsies we observed absent to low HLA-G expression on allograft epithelium in the presence of a donor CG or GG genotype at SNP +3142 . Therefore, our observations suggest that this genotype reduces the resistance of donor tissue to alloimmune attack (figure 2). Investigations on SNP -56(CC) and allele $\mathrm{G}^{*} 01: 03(\mathrm{AA})$ are limited $[7,13]$ and ours is the first report

\begin{tabular}{|c|c|c|c|}
\hline Variable & Hazard ratio $(95 \% \mathrm{CI})$ & p-value & $\mathbf{R}^{2}$-value \\
\hline SNP 201 (donor-recipient) CC-CT combination & $0.45(0.21-0.95)$ & 0.036 & 0.037 \\
\hline SNP 716 (donor-recipient) GT-TT combination & $0.45(0.21-0.95)$ & 0.036 & 0.037 \\
\hline
\end{tabular}

Adjusted for cytomegalovirus status. SNP: single nucleotide polymorphism. 
a)
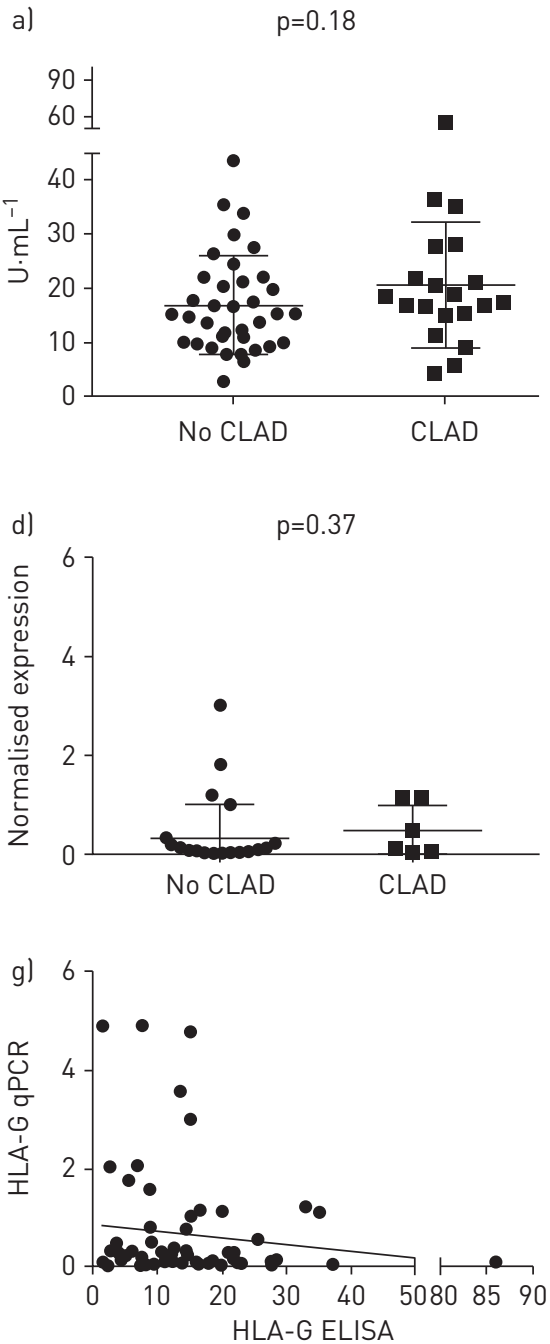

b)

$p=0.0497$

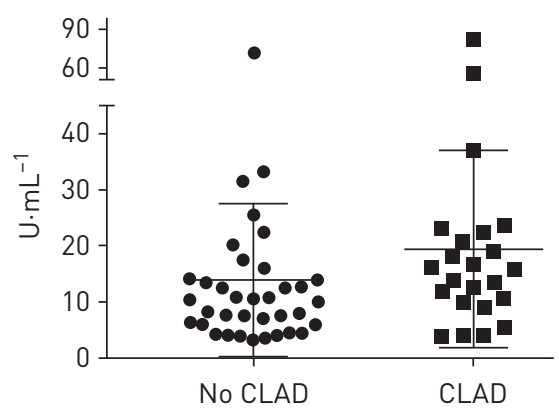

e)
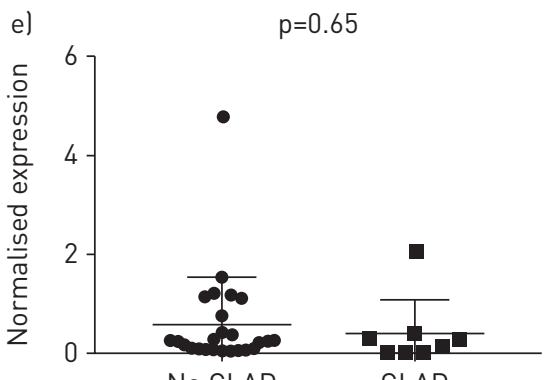

c)

$p=0.0326$
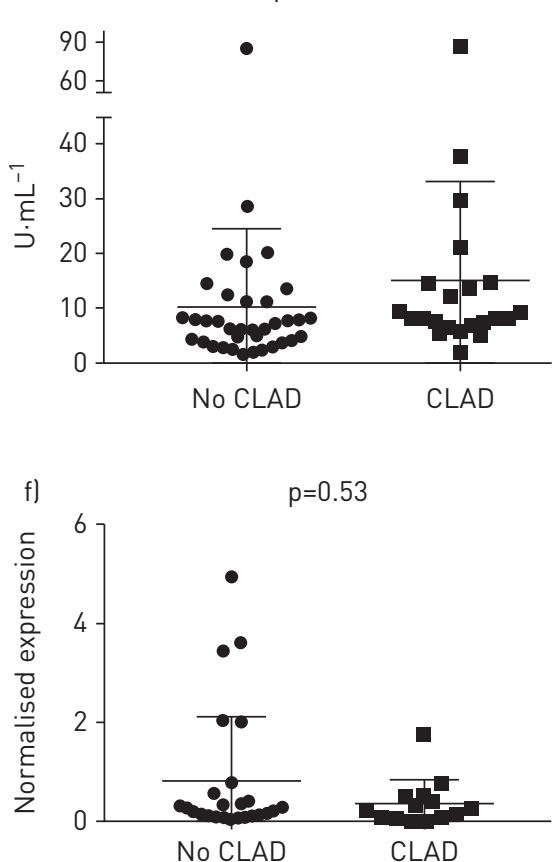

FIGURE 3 Bronchoalveolar lavage (BAL) supernatant soluble human leukocyte antigen-G (HLA-G) protein and cell pellet transcript abundance in recipients who remained chronic lung allograft dysfunction (CLAD)-free at 4 years (no $C L A D, n=43$ ) and those who developed CLAD within 36 months (CLAD, $n=25$ ). a-c) Measurement of soluble HLA-G in BAL supernatant by ELISA post transplant revealed median soluble HLA-G concentrations in patients with and without CLAD of a) 15.14 and $17.95 \mathrm{U} \cdot \mathrm{mL}^{-1}$ at 3 months, b) 10.45 and $15.81 \mathrm{U} \cdot \mathrm{mL}^{-1}$ at $6 \mathrm{months}$ and c) 6.65 and 8.04 at 9 months. Mann-Whitney p-values for the comparison between patients with and without CLAD are indicated. d-f) HLA-G transcripts from BAL cell pellets were examined by quantitative real-time PCR and normalised to the housekeeping gene peptidyl prolyl isomerase A (PPIA) at d) 3 months, e) 6 months and f) 9 months post transplant. Mann-Whitney p-values for the comparison between patients with and without CLAD are indicated. g) Comparison of HLA-G transcript abundance (y-axis) in BAL cell pellet with soluble HLA-G protein (x-axis) in BAL supernatant from the same BAL sample. $R^{2}=0.017, p=0.289$.

associating them with a protective effect when expressed in donor cells. Lastly, DEL allele in the $14 \mathrm{bp}$ INDEL polymorphism has been widely studied [7]. We found that this allele was associated with increased HLA-G expression and protection against acute cardiac allograft rejection [22]. Our observation that DEL alleles at $14 \mathrm{bp} \mathrm{INDEL} \mathrm{confer} \mathrm{a} \mathrm{reduced} \mathrm{risk} \mathrm{for} \mathrm{mortality} \mathrm{after} \mathrm{LT} \mathrm{is} \mathrm{also} \mathrm{in} \mathrm{keeping} \mathrm{with} \mathrm{this} \mathrm{finding.}$

Donor-recipient pairing (CC-CC) at SNP -201 was associated with cardiac allograft vasculopathy in our heart transplant cohort [17]. SNP -201 is close to a regulatory factor binding site that alters HLA-G transcription [17, 23]. Whereas SNP -201(CC) was associated with a poor outcome after heart transplantation, it has an opposite effect in LT (tables 2 and 3). This is not the first time that one HLA-G allele has been associated with opposite outcomes in different settings, suggesting distinct underlying mechanisms of protection conferred by HLA-G in different contexts [24]. Whereas cardiac allografts are fully contained within the thorax, transplanted lungs are exposed via the airways to inflammatory stimuli $[3,25]$. Thus, we speculate that there could be an interaction between the environment and donor SNP -201 that alters its relationship to graft outcome. Finally, SNP -716 C allele has been linked with lower HLA-G expression [15], and we found that the $\mathrm{T}$ allele at this locus in the donor confers reduced mortality and CLAD risk. This observation further supports the notion that donor HLA-G expression is a 
TABLE 4 Multivariable analysis of HLA-G as time-dependent variable for time to chronic lung allograft dysfunction

\begin{tabular}{lcc} 
Variable & Hazard ratio $(95 \% \mathrm{CI})$ & p-value \\
\hline HLA-G BAL levery 10 units $\left.\cdot \mathrm{mL}^{-1}\right)^{\#}$ & $1.20(1.01-1.43)$ & 0.04 \\
CMV (D+R-) & $2.11(0.96-4.64)$ & 0.06 \\
\hline
\end{tabular}

Multivariable analysis included the variable CMV status, which was significant in the univariate analysis. HLA-G: human leukocyte antigen-G; BAL: bronchoalveolar lavage; CMV: cytomegalovirus; D: donor; R: recipient. " : with imputation of missing values, hazard ratio 1.22 (95\% Cl 1.02-1.45; $\mathrm{p}=0.03$ ).

determinant of outcome after LT. Given these observations, it is not surprising that we detected significant levels of HLA-G expression in otherwise quiescent lung allograft tissue (figure $4 \mathrm{a}-\mathrm{c}$ ). Taken together, our findings indicate that donor SNPs which have, in other contexts, been associated with higher levels of HLA-G expression are associated with reduced mortality after LT.

Our data implicate HLA-G expression by donor tissue as a modulator of long-term outcome. Surprisingly, although it is known that allograft parenchymal cells express HLA-G, previous analyses have been restricted to recipient genotypes [15, 26] and thus the role of the donor HLA-G genotype was unknown [7]. Our previous heart transplant study was the first to examine donor and recipient HLA-G SNPs, revealing that specific combinations of donor and recipient alleles influenced outcome [17]. Our current findings corroborate and support this notion in LT.
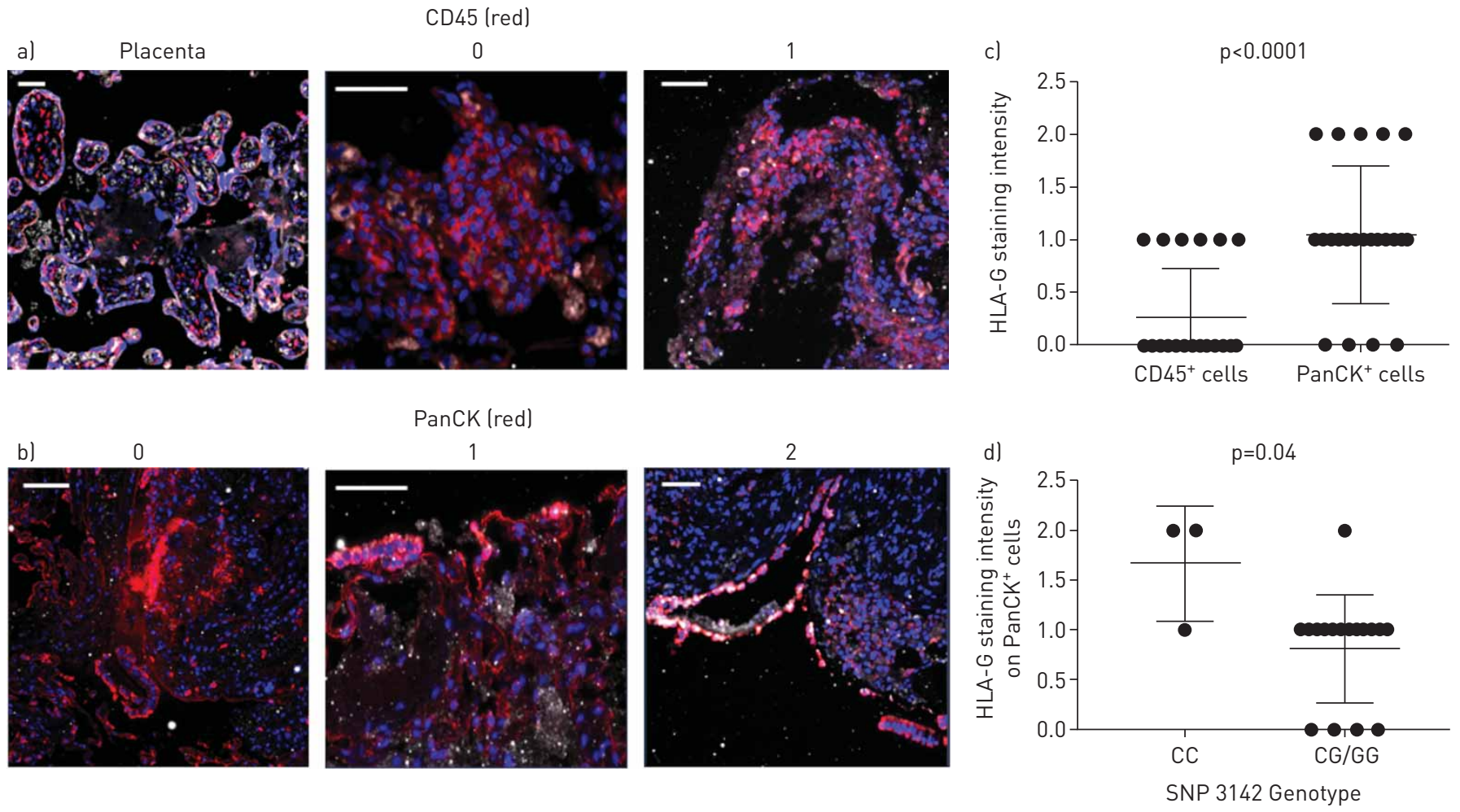

FIGURE 4 Predominantly epithelial human leukocyte antigen-G (HLA-G) expression in transbronchial biopsies (TBBx) without evidence of rejection ( $n=22$ patients). a, b) Paraffin-embedded placenta or TBBx samples were stained with DAPI (blue) and antibodies to HLA-G (white) and either a) CD45 (red) or b) pan-cytokeratin (PanCK) (red). Placental tissue stained for HLA-G (positive control) and CD45 is shown on the left of a. HLA-G staining was graded from 0 (absent) to 2 (strongly expressed) on leukocytes (CD45) and epithelial cells $\left(\right.$ PanCK $\left.{ }^{+}\right)$. Scale bars represent $50 \mu \mathrm{m}$. c) HLA-G expression was higher in epithelial cells than leukocytes in the same TBBx sample $(n=22)$. Wilcoxon matched pairs test $p<0.0001$. d) Epithelial staining intensity according to donor single nucleotide polymorphism (SNP) +3142 genotype (CC ( $n=3$ ) versus CG/GG ( $n=16$ ); donor genotype was unavailable for three). Mann-Whitney test $p=0.04$. For $c$ and $d$, each data point represents the mean value of 10 fields selected randomly using the DAPI channel. 
To examine how HLA-G expression in the graft might influence LT outcome, we measured HLA-G in serial BAL supernatants and cell pellets, which demonstrated that higher levels of sHLA-G at 6 and 9 months post transplant were seen in patients with subsequent CLAD. In a similar vein, BURGIERE et al. [16] observed higher serum concentrations of HLA-G at 12 months post transplant in patients who went on to develop CLAD. In contrast, previous transplantation studies revealed that higher levels of sHLA-G in serum conferred a reduced risk [11, 12]. However, White et al. [14] also observed that elevated sHLA-G in BAL fluid was associated with acute rejection and CLAD. BAL fluid contains many dead leukocytes and epithelial cells [27], and because the release of sHLA-G is a post-translational process that may not directly reflect the level of gene transcription [5], we speculate that increased sHLA-G in the BAL fluid is a reactive process that portends a detrimental long-term outcome. In keeping with this assertion, HLA-G gene expression in BAL cell pellets bore no relationship to sHLA-G in the same sample, nor did it impact on long-term graft outcome. It is important to note, however, that sHLA-G may exert important immunomodulatory effects in other compartments and contexts $[8,15]$.

In TBBx without evidence of rejection, we found that HLA-G expression was stronger in epithelial cells (donor-derived) than in leukocytes (recipient-derived) in the same biopsy. These observations imply that HLA-G expression is strongest in donor-derived cells, supporting our finding that donor HLA-G SNPs influence LT outcome. Additionally, donor SNP +3142 (CC) allele was associated with higher epithelial HLA-G expression in the small subset we examined, and was also associated with reduced mortality. We speculate that donor HLA-G SNPs control allograft epithelial HLA-G expression and that this is a mechanism through which HLA-G influences LT outcome [7]. It seems likely that HLA-G SNPs are of varying importance in different contexts $[22,24]$, which may help to explain why the same SNPs had different impacts in cardiac [17] (no epithelial cells) and lung (many epithelial cells) allografts. Graft chimerism, in which donor and recipient epithelial cells co-populate the lung allograft, may add an additional layer of complexity to this analysis [28]. It should be noted, however, that recipient epithelial chimerism in human lung allografts has been reported at less than $10 \%[29,30]$. The summative expression of HLA-G by donor and recipient cells at appropriate sites and times is likely to determine its overall impact [7]. Our study reveals that ignoring donor HLA-G SNPs disregards an important factor altering the expression of this molecule in LT.

Our study has important limitations. Panel-reactive antibody and HLA antibody data were missing prior to 2008, restricting our ability to assess the full impact of pre-transplant sensitisation on this cohort. Further, our BAL and TBBx samples were limited, meaning that analysis of these specimens may have been underpowered. Some limitations of our prior heart transplant study [17] also apply here. For instance, the available HLA-G ELISA kit only detects two of the seven known HLA-G isoforms [13]; it also cannot discern different HLA-G multimers, which is an important concern given that dimers more powerfully regulate inflammation than monomers [31]. Elevations in serum sHLA-G seem to be protective [22], whereas our results and those of WHITE et al. [14] indicate that elevations in BAL sHLA-G are deleterious. Unfortunately, we did not have access to serum samples from this cohort.

We did not have adequate power to analyse the effects of HLA-G haplotypes [32]. Di Cristofaro et al. [15] found that recipient haplotype UTR-3 was a risk factor for CLAD and was associated with lower serum sHLA-G in LT recipients. Further, HLA-G SNPs may act indirectly via linkage disequilibrium with other HLA polymorphisms [33, 34], which may themselves modify the risk of allograft rejection. Moreover, a recent report has explored polymorphisms in HLA-G, LILRB1/2 and KIR2DL4 genes and how SNPs in these genes affected susceptibility to and severity of endometriosis [35]. This area merits further study in solid organ transplantation.

With further validation, our results have important implications for LT. We recently demonstrated the feasibility of avoiding high-risk HLA mismatches in heart and lung recipients to prevent the development of de novo donor-specific antibody formation [36]. One could envision a similar approach to promote favourable HLA-G pairing between donors and recipients, although this strategy would not be applicable in the short term. Future research should also focus on establishing the mechanisms that link HLA-G SNPs with HLA-G expression in the allograft, because such approaches may uncover important therapeutic strategies for those with unfavourable HLA-G donor or recipient SNPs. Our findings add to the growing body of research on HLA-G and highlight the novel role of the donor HLA-G genotype in post-LT outcome.

Acknowledgements: The authors wish to thank Amsale Belay from the Clinical Genomics Centre at Sinai Health System for HLA-G SNP sequencing, Martin Oberle for assistance with samples in the TLTP biobank, and Hallie Ross and David Hwang for assistance with archival TBBx.

Author contributions: J. Lazarte conceived of the project, collected data, performed ELISA, analysed data and wrote the manuscript. S. Azad collected data. J. Ma and G. Tomlinson assisted with project design and performed statistical 
analyses. M. White, Z. Guan and J. Pelling performed immunofluorescence and quantitative PCR experiments and analysed data. T. Martinu, W. Klement and J. Tikkanen performed analysis of CLAD data. L. Levy performed analysis of PCR and ELISA data with reference to CLAD. V. Rao, D. Delgado and S. Keshavjee conceived of the project and contributed to study design. T. Martinu contributed to study design and overall data analysis. S.C. Juvet conceived of the project, collected and analysed PCR, ELISA and CLAD data, supervised data collection, and wrote the manuscript.

Conflict of interest: J. Lazarte has nothing to disclose. J. Ma has nothing to disclose. T. Martinu has nothing to disclose. L. Levy has nothing to disclose. W. Klement has nothing to disclose. M. White has nothing to disclose. J. Pelling has nothing to disclose. Z. Guan has nothing to disclose. S. Azad has nothing to disclose. J. Tikkanen has nothing to disclose. V. Rao reports personal fees for advisory board work from Medtronic and personal fees for consultancy from Abbott, outside the submitted work. G. Tomlinson has nothing to disclose. D. Delgado has nothing to disclose S. Keshavjee is a founder of Perfusix Canada, a non-profit company that provides ex vivo lung perfusion services to the University Health Network (Perfusix played no role in the conduct of this study); and is a founder of XOR Labs, Toronto, a company currently developing a medical device for ex vivo lung perfusion (not used in this study). S.C. Juvet has nothing to disclose.

Support statement: J. Lazarte was supported by an International Society for Heart and Lung Transplantation (ISHLT) travel award. The work was supported by institutional start-up funds (to S.C. Juvet) and by Canadian Institutes for Health Research Project Bridge Grant (to S.C. Juvet and T. Martinu). Funding information for this article has been deposited with the Crossref Funder Registry.

\section{References}

1 Yusen RD, Edwards LB, Kucheryavaya AY, et al. The registry of the International Society for Heart and Lung Transplantation: thirty-first adult lung and heart-lung transplant report-2014; focus theme: retransplantation. J Heart Lung Transplant 2014; 33: 1009-1024.

2 Sato M, Waddell TK, Wagnetz U, et al. Restrictive allograft syndrome (RAS): a novel form of chronic lung allograft dysfunction. J Heart Lung Transplant 2011; 30: 735-742.

3 Royer PJ, Olivera-Botello G, Koutsokera A, et al. Chronic lung allograft dysfunction: a systematic review of mechanisms. Transplantation 2016; 100: 1803-1814.

4 Verleden SE, Todd JL, Sato M, et al. Impact of CLAD phenotype on survival after lung retransplantation: a multicenter study. Am J Transplant 2015; 15: 2223-2230.

5 Paul P, Cabestre FA, Ibrahim EC, et al. Identification of HLA-G7 as a new splice variant of the HLA-G mRNA and expression of soluble HLA-G5, -G6, and -G7 transcripts in human transfected cells. Hum Immunol 2000; 61: 1138-1149.

6 Shiroishi M, Kuroki K, Ose $\mathrm{T}$, et al. Efficient leukocyte Ig-like receptor signaling and crystal structure of disulfide-linked HLA-G dimer. J Biol Chem 2006; 281: 10439-10447.

7 Carosella ED, Rouas-Freiss N, Roux DT, et al. HLA-G: an immune checkpoint molecule. Adv Immunol 2015; 127: 33-144.

8 Brugiere $\mathrm{O}$, Thabut G, Pretolani M, et al. Immunohistochemical study of HLA-G expression in lung transplant recipients. Am J Transplant 2009; 9: 1427-1438.

9 Shiroishi M, Tsumoto K, Amano K, et al. Human inhibitory receptors Ig-like transcript 2 (ILT2) and ILT4 compete with CD8 for MHC class I binding and bind preferentially to HLA-G. Proc Natl Acad Sci USA 2003; 100: $8856-8861$

10 Rebmann V, da Silva Nardi F, Wagner B, et al. HLA-G as a tolerogenic molecule in transplantation and pregnancy. J Immunol Res 2014; 2014: 297073.

11 Lila N, Amrein C, Guillemain R, et al. Human leukocyte antigen-G expression after heart transplantation is associated with a reduced incidence of rejection. Circulation 2002; 105: 1949-1954.

12 Creput C, Durrbach A, Menier C, et al. Human leukocyte antigen-G (HLA-G) expression in biliary epithelial cells is associated with allograft acceptance in liver-kidney transplantation. J Hepatol 2003; 39: 587-594.

13 Donadi EA, Castelli EC, Arnaiz-Villena A, et al. Implications of the polymorphism of HLA-G on its function, regulation, evolution and disease association. Cell Mol Life Sci 2011; 68: 369-395.

14 White SR, Floreth T, Liao C, et al. Association of soluble HLA-G with acute rejection episodes and early development of bronchiolitis obliterans in lung transplantation. PLoS One 2014; 9: e103643.

15 Di Cristofaro J, Reynaud-Gaubert M, Carlini F, et al. HLA-G*01:04 UTR3 recipient correlates with lower survival and higher frequency of chronic rejection after lung transplantation. Am I Transplant 2015; 15: 2413-2420.

16 Brugiere O, Thabut G, Krawice-Radanne I, et al. Role of HLA-G as a predictive marker of low risk of chronic rejection in lung transplant recipients: a clinical prospective study. Am J Transplant 2015; 15: 461-471.

17 Lazarte J, Goldraich L, Manlhiot C, et al. Human leukocyte antigen G single-nucleotide polymorphism -201 (CC-CC) donor-recipient genotype matching as a predictor of severe cardiac allograft vasculopathy. J Heart Lung Transplant 2016; 35: 1101-1107.

18 Tinckam KJ, Keshavjee S, Chaparro C, et al. Survival in sensitized lung transplant recipients with perioperative desensitization. Am J Transplant 2015; 15: 417-426.

19 Klement W, Tikkanen J, Juvet S, et al. Automating the diagnosis of potential CLAD and its subtypes in lung transplant recipients. J Heart Lung Transpl 2017; 36: S309-S309.

20 Meyer KC, Raghu G, Verleden GM, et al. An international ISHLT/ATS/ERS clinical practice guideline: diagnosis and management of bronchiolitis obliterans syndrome. Eur Respir J 2014; 44: 1479-1503.

21 Rizzo R, Bortolotti D, Ben Fredj N, et al. Role of HLA-G 14 bp deletion/insertion and $+3142 \mathrm{C}>\mathrm{G}$ polymorphisms in the production of sHLA-G molecules in relapsing-remitting multiple sclerosis. Hum Immunol 2012; 73: $1140-1146$.

22 Twito T, Joseph J, Mociornita A, et al. The 14-bp deletion in the HLA-G gene indicates a low risk for acute cellular rejection in heart transplant recipients. J Heart Lung Transplant 2011; 30: 778-782.

23 Castelli EC, Veiga-Castelli LC, Yaghi L, et al. Transcriptional and posttranscriptional regulations of the HLA-G gene. J Immunol Res 2014; 2014: 734068. 
24 Hou W, Huang Z, Ji Z, et al. Human leukocyte antigen-G-14-base-pair-insertion/deletion polymorphism and graft survival in kidney transplant recipients. Exp Clin Transplant 2014; 12: 89-94.

25 Benmerad M, Slama R, Botturi K, et al. Chronic effects of air pollution on lung function after lung transplantation in the Systems prediction of Chronic Lung Allograft Dysfunction (SysCLAD) study. Eur Respir J 2017; 49: 1600206 .

26 Creput C, Le Friec G, Bahri R, et al. Detection of HLA-G in serum and graft biopsy associated with fewer acute rejections following combined liver-kidney transplantation: possible implications for monitoring patients. Hum Immunol 2003; 64: 1033-1038.

27 Levy L, Juvet SC, Boonstra K, et al. Sequential broncho-alveolar lavages reflect distinct pulmonary compartments: clinical and research implications in lung transplantation. Respir Res 2018; 19: 102.

28 Starzl TE, Demetris AJ, Trucco M, et al. Cell-migration and chimerism after whole-organ transplantation - the basis of graft acceptance. Hepatology 1993; 17: 1127-1152.

29 Kleeberger W, Versmold A, Rothamel T, et al. Increased chimerism of bronchial and alveolar epithelium in human lung allografts undergoing chronic injury. Am J Pathol 2003; 162: 1487-1494.

30 Spencer H, Rampling D, Aurora P, et al. Transbronchial biopsies provide longitudinal evidence for epithelial chimerism in children following sex mismatched lung transplantation. Thorax 2005; 60: 60-62.

31 Ezeakile M, Portik-Dobos V, Wu J, et al. HLA-G dimers in the prolongation of kidney allograft survival. J Immunol Res 2014; 2014: 153981.

32 Di Cristofaro J, El Moujally D, Agnel A, et al. HLA-G haplotype structure shows good conservation between different populations and good correlation with high, normal and low soluble HLA-G expression. Hum Immunol 2013; 74: 203-206.

33 Kirszenbaum M, Moreau P, Gluckman E, et al. An alternatively spliced form of HLA-G mRNA in human trophoblasts and evidence for the presence of HLA-G transcript in adult lymphocytes. Proc Natl Acad Sci USA 1994; 91: 4209-4213.

34 Morandi F, Pistoia V. Interactions between HLA-G and HLA-E in physiological and pathological conditions. Front Immunol 2014; 5: 1-6.

35 Bylinska A, Wilczynska K, Malejczyk J, et al. The impact of HLA-G, LILRB1 and LILRB2 gene polymorphisms on susceptibility to and severity of endometriosis. Mol Genet Genomics 2018; 293: 601-613.

36 Zamel R, McCaughan J, Hirji A, et al. Feasibility of avoiding higher risk epitope and allele hla mismatch to reduce de novo donor specific antibody (dnDSA) in lung transplantation. J Heart Lung Transpl 2018; 37: S17-S18. 